PROCEEDINGS OF THE

AMERICAN MATHEMATICAL SOCIETY

Volume 129, Number 11, Pages 3313-3320

S 0002-9939(01)05941-X

Article electronically published on April 9, 2001

\title{
A NOTE ON EXTENSIONS OF ASYMPTOTIC DENSITY
}

\author{
A. BLASS, R. FRANKIEWICZ, G. PLEBANEK, AND C. RYLL-NARDZEWSKI
}

(Communicated by Dale Alspach)

\begin{abstract}
By a density we mean any extension of the asymptotic density to a finitely additive measure defined on all sets of natural numbers. We consider densities associated to ultrafilters on $\omega$ and investigate two additivity properties of such densities. In particular, we show that there is a density $\nu$ for which $L_{1}(\nu)$ is complete.
\end{abstract}

\section{INTRODUCTION}

We denote the set of natural numbers by $\omega(=\{0,1,2, \ldots\})$, and often regard any $n \in \omega$ as the set $\{0,1, \ldots, n-1\}$. The symbol $\mathcal{P}(\omega)$ stands for the family of all subsets of $\omega$. Recall that the asymptotic density of a set $A \subseteq \omega$, denoted here by $d(A)$, is defined as

$$
d(A)=\lim _{n \rightarrow \infty} \frac{|A \cap n|}{n},
$$

provided this limit exists. Note that, while $d$ is finitely additive, the domain of $d$ is not an algebra. Several authors have considered extensions of asymptotic density to a finitely additive measure defined on a certain algebra of sets; see for instance Buck [2], Maharam [6], and Mekler [7]. In the sequel, any finitely additive $\nu$ defined on $\mathcal{P}(\omega)$ and extending $d$ will be called a density.

Following Buck [2] and Mekler [7], we shall consider a certain additivity property of densities. Say that a density $\nu$ has property $\mathbf{A P}(*)$ if for every increasing sequence $\left(A_{i}\right)_{i \geq 1} \subseteq \mathcal{P}(\omega)$ there is a set $B \in \mathcal{P}(\omega)$ such that

(i) $A_{i} \subseteq^{*} B$ for every $i$;

(ii) $\nu(B)=\lim _{i} \nu\left(A_{i}\right)$.

Here and below, we write $A \subseteq^{*} B$ to denote that the set $A \backslash B$ is finite; in this case we also say that $A$ is almost included in $B$.

Suppose now that a $\sigma$-algebra $\mathcal{F}$ of subsets of an arbitrary space $X$ is given, and that $\nu$ is a finitely additive finite measure defined on $\mathcal{F}$. One can consider the following natural weakening of $\mathbf{A P}(*)$ : Say that $\nu$ has property $\mathbf{A P}($ null) if for every increasing sequence $\left(A_{i}\right)_{i \geq 1} \subseteq \mathcal{F}$ there is a set $B \in \mathcal{F}$ such that

(i) ${ }^{\prime} \nu\left(A_{i} \backslash B\right)=0$ for every $i$;

(ii) $\nu(B)=\lim _{i} \nu\left(A_{i}\right)$.

Received by the editors June 29, 1999 and, in revised form, March 17, 2000.

2000 Mathematics Subject Classification. Primary 28A12; Secondary 03E05, 03E35, 11B05.

The first-named author was partially supported by NSF grant DMS-9505118.

The other authors were partially supported by KBN grant 2P03A 01813. 
Property $\mathbf{A P}$ (null), which is a weak version of continuity from below, characterizes those finitely additive measures $\nu$ (defined on a $\sigma$-algebra $\mathcal{F}$ ), for which the space $L_{1}(\nu)$ is complete in the "usual" metric; see [5] and [4] (and see [1] for the theory of $L_{1}$ spaces over finitely additive measures).

The authors of [5] asked whether there exists a density with property $\mathbf{A P}$ (null). We give below a positive answer to that question and present some related results, some of which build on ideas from [7].

Every free ultrafilter $\mathcal{U}$ on $\omega$ defines, in a natural way, a certain density $\nu_{\mathcal{U}}$ (see section 2). We shall show that $\nu_{\mathcal{U}}$ has property $\mathbf{A P}($ null) whenever $\mathcal{U}$ contains a set which is thin enough. Our next result yields a short proof of a result due to Mekler [7]-we show that $\nu_{\mathcal{U}}$ has property $\mathbf{A P}\left(^{*}\right)$ provided $\mathcal{U}$ is a $\mathrm{P}$-point ultrafilter. We also prove that there is a $\mathcal{U}$ giving a density $\nu_{\mathcal{U}}$ which has property $\mathbf{A P}$ (null) but fails to have $\mathbf{A P}(*)$. Finally, we explain that, roughly speaking, one cannot retrieve properties of $\mathcal{U}$ from corresponding properties of $\nu_{\mathcal{U}}$. In particular, $\nu_{\mathcal{U}}$ can have property $\mathbf{A P}\left(^{*}\right)$ even if $\mathcal{U}$ is not a $\mathrm{P}$-point.

\section{Ultrafilters AND DENSITIES}

Let $\mathcal{U}$ be a free ultrafilter on a space $X$. Given a bounded function $\alpha: X \rightarrow \mathbf{R}$, we write $a=\mathcal{U}-\lim _{x} \alpha(x)$ if $\left\{x:\left|a_{x}-a\right|<\varepsilon\right\} \in \mathcal{U}$ for every positive $\varepsilon$. If $\mathcal{U}$ is a free ultrafilter on $\omega$, then it is routine to check that the formula

$$
\nu_{\mathcal{U}}(A)=\mathcal{U}-\lim _{n} \frac{|A \cap n|}{n},
$$

defines a finitely additive extension of the asymptotic density to the power set of $\omega$ (that is, $\nu \mathcal{U}$ is a density in our terminology). The averaging process involved in densities can also be expressed in terms of the Cesàro matrix, as explained in [7].

Recall that a free ultrafilter $\mathcal{U}$ is called a $P$-point if for every sequence $\left(X_{i}\right)$ of elements of $\mathcal{U}$ one can find a set $Y \in \mathcal{U}$ which is almost contained in every $X_{i}$. It is well-known that P-points do exist under Martin's axiom; however, their nonexistence is also relatively consistent (see [8, Section VI.4]).

Given an infinite set $X \subseteq \omega$, we write

$$
I_{n}^{X}=[\max (X \cap n), n) \cap \omega,
$$

whenever $n \in X$. Say that a set $X$ is thin if

$$
\lim _{n \in X} \frac{\left|I_{n}^{X}\right|}{n}=1
$$

In other words, a set $X$ is thin if, enumerating $X$ as $\left(n_{k}\right)_{k}$ in increasing order, we have $\lim _{k} n_{k} / n_{k+1}=0$.

Lemma 1. If an ultrafilter $\mathcal{U}$ contains a thin set $X$, then

$$
\nu_{\mathcal{U}}(A)=\mathcal{U}-\lim _{n} \frac{\left|A \cap I_{n}^{X}\right|}{n}=\mathcal{U}-\lim _{n} \frac{\left|A \cap I_{n}^{X}\right|}{\left|I_{n}^{X}\right|},
$$

for every set $A$.

Proof. The second equation is obvious as $X$ is thin and in $\mathcal{U}$. For the first equation, notice that

$$
A \cap I_{n}^{X} \subseteq A \cap n \subseteq\left(A \cap I_{n}^{X}\right) \cup \max (X \cap n) .
$$


Therefore,

$$
\frac{\left|A \cap I_{n}^{X}\right|}{n} \leq \frac{|A \cap n|}{n} \leq \frac{\left|A \cap I_{n}^{X}\right|}{n}+\frac{n-\left|I_{n}^{X}\right|}{n} .
$$

Taking the limit along $\mathcal{U}$ and remembering that $X \in \mathcal{U}$ is thin, we obtain the first equation of the lemma.

Theorem 1. If an ultrafilter $\mathcal{U}$ contains a thin set $X$, then $\nu_{\mathcal{U}}$ is a density having property $\mathbf{A P}$ (null).

Proof. Having an ultrafilter $\mathcal{U}$ and a thin set $X \in \mathcal{U}$ fixed, we write $\nu=\nu_{\mathcal{U}}$ and $I_{n}=I_{n}^{X}$ for simplicity. By Lemma 1 we have $\nu(A)=\mathcal{U}$ - $\lim _{n} \frac{\left|A \cap I_{n}\right|}{n}$ for every $A$.

To check $\mathbf{A P}$ (null) take an increasing sequence $\left(A_{i}\right)_{i \geq 1}$ and put $\alpha=\lim _{i} \nu\left(A_{i}\right)$. Passing to a subsequence we may assume that $\nu\left(A_{i}\right) \geq \alpha-1 / i$.

Find a decreasing sequence $\left(X_{i}\right)_{i \geq 1}$ of elements of $\mathcal{U}$ such that $X_{1} \subseteq X, X_{i} \cap i=$ $\emptyset$, and

$$
\left|\frac{\left|A_{i} \cap I_{n}\right|}{\left|I_{n}\right|}-\nu\left(A_{i}\right)\right|<\frac{1}{i}
$$

whenever $n \in X_{i}$.

Now we define a set $B$, separately on each segment $I_{n}$. Put $B \cap I_{n}=\emptyset$ for $n \in X \backslash X_{1}$ and $B \cap I_{n}=A_{i} \cap I_{n}$ for $n \in X_{i} \backslash X_{i+1}$.

Let $n \in X_{i}$; then $n \in X_{j} \backslash X_{j+1}$ for some $j \geq i$. It follows that

$$
\alpha-\frac{2}{i} \leq \nu\left(A_{j}\right)-\frac{1}{j} \leq \frac{\left|B \cap I_{n}\right|}{\left|I_{n}\right|}=\frac{\left|A_{j} \cap I_{n}\right|}{\left|I_{n}\right|} \leq \nu\left(A_{j}\right)+\frac{1}{j} \leq \alpha+\frac{1}{i},
$$

and hence for every $n \in X_{i}$ we have

$$
\left|\frac{\left|B \cap I_{n}\right|}{\left|I_{n}\right|}-\alpha\right|<\frac{2}{i}
$$

which gives $\nu(B)=\alpha$.

Now we check that $\nu\left(A_{i} \backslash B\right)=0$. Indeed, if $n \in X_{i}$, then for some $j \geq i$ we have $B \cap I_{n}=A_{j} \cap I_{n} \supseteq A_{i} \cap I_{n}$. Thus $\left(A_{i} \backslash B\right) \cap I_{n}=\emptyset$ for every $n \in X_{i}$, and we are done.

We next give a short proof of Mekler's result [7] that a P-point yields a density with $\mathbf{A P}(*)$.

Theorem 2. If $\mathcal{U}$ is a P-point ultrafilter, then $\nu_{\mathcal{U}}$ is a density having property $\mathbf{A P}(*)$.

Proof. For an increasing sequence $\left(A_{i}\right)_{i}$ we take sets $X_{i}$ belonging to $\mathcal{U}$ and such that

$$
\left|\frac{\left|A_{i} \cap n\right|}{n}-\nu\left(A_{i}\right)\right|<\frac{1}{i}
$$

whenever $n \in X_{i}$. As in the proof of Theorem 1, we write $\alpha=\lim _{i} \nu\left(A_{i}\right)$. Now, since $\mathcal{U}$ is a P-point, there is a set $Y \in \mathcal{U}$ such that $Y \subseteq^{*} X_{i}$ for every $i$. Choose an increasing sequence of numbers $m_{i} \in \omega$ so that $Y \subseteq X_{i} \cup m_{i}$.

We define a set $B$ so that $B \cap\left[m_{i}, m_{i+1}\right)=A_{i} \cap\left[m_{i}, m_{i+1}\right)$ for every $i$. We have $A_{i} \subseteq^{*} B$, since $A_{i} \backslash B \subseteq m_{i}$. If $n \in\left[m_{i}, m_{i+1}\right)$, then $B \cap n \subseteq A_{i} \cap n$. If, moreover, $n \in Y$, then $n \in X_{i}$ so

$$
\frac{|B \cap n|}{n} \leq \frac{\left|A_{i} \cap n\right|}{n}<\nu\left(A_{i}\right)+\frac{1}{i} \leq \alpha+\frac{1}{i} .
$$


It follows that the inequality above holds for every $n \in Y \backslash m_{i}$, and therefore $\nu_{\mathcal{U}}(B) \leq \alpha$. On the other hand, the reverse inequality holds because $\nu_{\mathcal{U}}(B) \geq$ $\nu_{\mathcal{U}}\left(A_{i}\right)$ for every $i$.

Theorem 3. Suppose that $\mathcal{U}$ is an ultrafilter containing a thin set. Then $\nu_{\mathcal{U}}$ has property $\mathbf{A P}(*)$ if and only if $\mathcal{U}$ is a P-point.

Proof. One direction is Theorem 2 (and doesn't need thinness). To prove the other direction, fix a thin set $X$ belonging to $\mathcal{U}$; again let $I_{n}$ stand for $I_{n}^{X}$. Let $\left(X_{m}\right)_{m}$ be a decreasing sequence in $\mathcal{U}$. For every $m$ write

$$
A_{m}=\bigcup_{k \in X \backslash X_{m}} I_{k}
$$

Then $A_{m} \cap I_{n}=\emptyset$ for every $n \in X_{m}$, and Lemma 1 gives $\nu_{\mathcal{U}}\left(A_{m}\right)=0$.

Since $\nu_{\mathcal{U}}$ has property $\mathbf{A P}(*)$, there is a set $A$ with $\nu_{\mathcal{U}}(A)=0$, and such that $A_{m} \subseteq^{*} A$ for every $m$. It follows that

$$
Y=\left\{k: \frac{\left|A \cap I_{k}\right|}{\left|I_{k}\right|}<\frac{1}{2}\right\} \in \mathcal{U}
$$

Since $A_{m}$ is almost contained in $A$, we have $A_{m} \subseteq A \cup k_{m}$ for some $k_{m} \in X$. Then $Y \subseteq X_{m} \cup k_{m}$. Indeed, if $k \in Y \backslash X_{m}$, then

$$
\frac{\left|A \cap I_{k}\right|}{\left|I_{k}\right|}<\frac{1}{2} \quad \text { and } \quad A_{m} \cap I_{k}=I_{k} .
$$

Hence $A_{m} \cap I_{k} \nsubseteq \subseteq A \cap I_{k}$, which means $k \leq k_{m}$. The proof is complete.

In connection with Theorem 3 it is perhaps worth remarking that, assuming Martin's axiom, one can easily construct a P-point which does not contain any thin set.

Given an arbitrary (infinite) thin set $X$, it is easy to find a free ultrafilter $\mathcal{U}$ which contains $X$ but is not a P-point (take a sequence if distinct ultrafilters containing $X$ and let $\mathcal{U}$ be its cluster point). In view of Theorem 2 and Theorem 3 , this remark yields the following.

Corollary 4. There exists a free ultrafilter $\mathcal{U}$ such that $\nu_{\mathcal{U}}$ has property $\mathbf{A P}$ (null) but not property $\mathbf{A P}(*)$.

\section{Different ultrafilters giving the same Density}

Extending an idea suggested by Example 1.5 of [7], we shall show that rather dissimilar ultrafilters $\mathcal{U}$ can lead to similar densities or even the same density.

If $\mathcal{U}$ is an ultrafilter on a set $X$ and $g: X \longrightarrow Y$ is any mapping, then $g(\mathcal{U})$ denotes the ultrafilter on $Y$ consisting of those $B \subseteq Y$ for which $g^{-1}(B) \in \mathcal{U}$. Note that

$$
g(\mathcal{U})-\lim _{y} \alpha(y)=\mathcal{U}-\lim _{x} \alpha(g(x)),
$$

for every function $\alpha$ from $Y$ into $[0,1]$.

We consider the set $\Delta=\{(n, k): n<k\}$ and "the standard pairing function" $p: \Delta \longrightarrow \omega$, where

$$
p(n, k)=\frac{k(k-1)}{2}+n .
$$


Put also $q(k)=p(0, k)$ and denote by $\pi: \Delta \longrightarrow \omega$ the projection onto the second coordinate.

Lemma 2. If $\mathcal{U}$ is a free ultrafilter on $\Delta$, then $\nu_{p(\mathcal{U})}=\nu_{q \circ \pi(\mathcal{U})}$.

Proof. (1) Note first that for any $A \subseteq \omega$ and natural numbers $Q, P$, if $Q \leq P \leq$ $Q+2 \sqrt{Q}$, then

$$
\left|\frac{|A \cap Q|}{Q}-\frac{|A \cap P|}{P}\right| \leq \frac{2}{\sqrt{Q}} .
$$

Indeed, (1) follows immediately from the following two inequalities:

$$
\begin{gathered}
0 \geq \frac{|A \cap Q|}{Q}-\frac{|A \cap P|}{Q}=-\frac{|A \cap(P \backslash Q)|}{Q} \geq-\frac{P-Q}{Q} \geq-\frac{2}{\sqrt{Q}} ; \\
0 \leq \frac{|A \cap P|}{Q}-\frac{|A \cap P|}{P}=|A \cap P|\left(\frac{1}{Q}-\frac{1}{P}\right)=\frac{|A \cap P|}{P} \cdot \frac{P-Q}{Q} \leq \frac{2}{\sqrt{Q}} .
\end{gathered}
$$

(2) For every $n<k$ we have

$$
q(k) \leq p(n, k) \leq q(k)+2 \sqrt{q(k)} .
$$

Indeed, the definitions of $p(n, k)$ and $q(k)$ make the first inequality trivial, and they imply that

$$
p(n, k)-q(k)=n \leq k-1 \leq \sqrt{2 q(k)} \leq 2 \sqrt{q(k)},
$$

which gives the second inequality.

(3) Using (2) and (1) (where $Q=q(k), P=p(n, k)$ ), we get for any $A \subseteq \omega$

$$
\begin{aligned}
& \nu_{p(\mathcal{U})}(A)=p(\mathcal{U})-\lim _{m} \frac{|A \cap m|}{m}=\mathcal{U}-\lim _{(n, k)} \frac{|A \cap p(n, k)|}{p(n, k)} \\
= & \mathcal{U}-\lim _{(n, k)} \frac{|A \cap q(k)|}{q(k)}=q \circ \pi(\mathcal{U}) \lim \frac{|A \cap m|}{m}=\nu_{q \circ \pi(\mathcal{U})}(A) .
\end{aligned}
$$

Theorem 5. (a) If there exist P-point ultrafilters on $\omega$, then there is an ultrafilter $\mathcal{V}$ which is not a P-point, and such that $\nu_{\mathcal{V}}$ has property $\mathbf{A P}\left(^{*}\right)$.

(b) There is an ultrafilter $\mathcal{V}$ which does not contain a thin set, and such that $\nu_{\mathcal{V}}$ has property $\mathbf{A P}($ null $)$ but not $\mathbf{A P}(*)$.

Proof. Let $\mathcal{U}_{1}$ and $\mathcal{U}_{2}$ be ultrafilters on $\omega$ and let $\mathcal{U}=\mathcal{U}_{1} \otimes \mathcal{U}_{2}$ denote their product. By definition, $D \in \mathcal{U}_{1} \otimes \mathcal{U}_{2}$ if $\left\{n: D_{\mid n} \in \mathcal{U}_{2}\right\} \in \mathcal{U}_{1}$, where $D_{\mid n}$ is defined to be $\{k:(n, k) \in D\}$. Note that $\Delta \in \mathcal{U}_{1} \otimes \mathcal{U}_{2}$, so we may consider the product ultrafilter as defined on $\Delta$. Note also that $\pi\left(\mathcal{U}_{1} \otimes \mathcal{U}_{2}\right)=\mathcal{U}_{2}$.

To check (a) take a P-point $\mathcal{U}_{2}$ and arbitrary $\mathcal{U}_{1}$, and consider $\mathcal{U}=\mathcal{U}_{1} \otimes \mathcal{U}_{2}$. Using Lemma 2, we get

$$
\nu_{p\left(\mathcal{U}_{1} \otimes \mathcal{U}_{2}\right)}=\nu_{q \circ \pi\left(\mathcal{U}_{1} \otimes \mathcal{U}_{2}\right)}=\nu_{q\left(\mathcal{U}_{2}\right)} .
$$

Since $q\left(\mathcal{U}_{2}\right)$ is a P-point, $\nu_{p(\mathcal{U})}$ has property $\mathbf{A P}(*)$. On the other hand, $\mathcal{U}$ and its isomorphic copy $p(\mathcal{U})$ are not P-points, for no element of $\mathcal{U}$ is almost contained in every $\Delta_{N}=\{(n, k) \in \Delta: n \geq N\}$.

We argue for (b) in a similar manner. Take an ultrafilter $\mathcal{U}_{2}$ which is not a P-point and contains a thin set. Then $q\left(\mathcal{U}_{2}\right)$ contains a thin set, so $\nu_{q\left(\mathcal{U}_{2}\right)}$ has property $\mathbf{A P}\left(\right.$ null). On the other hand, $q\left(\mathcal{U}_{2}\right)$ is not a P-point, so $\nu_{q\left(\mathcal{U}_{2}\right)}$ does not have property $\mathbf{A P}(*)$ by Theorem 3 . Now $p(\mathcal{U})$ defines the same density but it does 
not contain a thin set. Indeed, if $A \subseteq \omega$ and $p^{-1}(A) \in \mathcal{U}$, then there are $i, j \in A$ with $i<j<2 i$.

\section{Densities without additivity properties}

Recall that it is relatively consistent that no density has property $\mathbf{A P}\left({ }^{*}\right)$; see Mekler [7], where Shelah's argument from [8] is suitably adapted. Frankiewicz, Shelah, Zbierski [3] obtained a model of set theory, in which there are no $c c c$ P-sets in $\beta \omega \backslash \omega$. Let us note that this result improves Mekler's theorem. Indeed, every density $\nu$ defines the unique Radon measure $\widehat{\nu}$ on the compact space $\beta \omega \backslash \omega$. If $S$ denotes the support of $\widehat{\nu}$, then $S$ is clearly $c c c$. If, moreover, $\nu$ has property $\mathbf{A P}\left({ }^{*}\right)$ then $S$ is easily seen to be a P-set.

Corollary 4 above shows that no extra axioms are needed to find an ultrafilter $\mathcal{U}$ such that $\nu_{\mathcal{U}}$ fails to have $\mathbf{A P}\left({ }^{*}\right)$. We sketch here another, more constructive, argument for this fact.

(1) First note that there is a sequence $\left(A_{i}\right)$ of subsets of $\omega$ such that for every $k$ and $\varepsilon>0$ the system of inequalities

$$
\frac{\left|A_{i} \cap n\right|}{n}<\varepsilon, \quad i=1,2, \ldots, k, \quad \frac{\left|A_{k+1} \cap n\right|}{n} \geq \frac{1}{2},
$$

is satisfied for infinitely many $n$.

This may be proved by a Baire category argument but, as the referee remarked, it suffices to put $A_{i}=\left\{\left[n_{k}, n_{k+1}\right): k \in W_{i}\right\}$, where $\left(n_{k}\right)_{k}$ is an enumeration of a thin set, and $\left(W_{i}\right)_{i}$ is a partition of $\omega$ into infinite sets.

(2) Denote by $\mathcal{B}$ the family of all sets $B$ almost including $A_{i}$ for every $i$. Put

$$
X_{\varepsilon, i}=\left\{n: \frac{\left|A_{i} \cap n\right|}{n}<\varepsilon\right\} ; \quad Y_{B}=\left\{n: \frac{|B \cap n|}{n} \geq \frac{1}{2}\right\}
$$

for every $i$ and $\varepsilon>0$, and for every $B \in \mathcal{B}$.

(3) Note that the set

$$
X_{\varepsilon, 1} \cap X_{\varepsilon, 2} \cap \ldots \cap X_{\varepsilon, k} \cap Y_{B}
$$

is infinite for any $\varepsilon, k$, and every $B \in \mathcal{B}$. Since $Y_{B} \cap Y_{C} \supseteq Y_{B \cap C}$ for $B, C \in \mathcal{B}$, it follows that the family of all $X_{\varepsilon, i}$ and all $Y_{B}$ has the strong finite intersection property, and therefore is contained in some free ultrafilter $\mathcal{U}$. Now we have $\nu_{\mathcal{U}}\left(A_{i}\right)=0$ for every $i$, and $\nu_{\mathcal{U}}(B) \geq 1 / 2$ for all $B \in \mathcal{B}$, so $\nu_{\mathcal{U}}$ does not have property $\mathbf{A P}(*)$.

Of course, property $\mathbf{A P}$ (null) is much harder to destroy, and we do not know if this can be done by a suitable modification of the argument above. In response to our question whether there is an ultrafilter giving a density without property AP(null), David Fremlin presented the following result (in a letter of October, 1999).

Theorem 6 (Fremlin). Suppose that $\mathcal{U}$ is an ultrafilter on $\omega$ such that for every $A \in \mathcal{U}$ there is a $k>0$ with $A+k \in \mathcal{U}$. Write $\mathcal{V}=g(\mathcal{U})$, where $g: \omega \rightarrow \omega$, $g(n)=2^{n}$. Then the density $\nu \mathcal{\nu}$ does not have property $\mathbf{A P}($ null $)$.

Proof. Clearly $\nu \nu=\nu$, where $\nu$ is a density defined by the formula

$$
\nu(A)=\mathcal{U}-\lim _{n} \frac{\left|A \cap 2^{n}\right|}{2^{n}} .
$$


For every $k \in \omega$ we find a set $I_{k} \in \mathcal{U}$ such that $I_{k} \cap(k+1)=\emptyset$ and $|i-j|>k$ whenever $i, j \in I_{k}, i \neq j$. For every $k$ we put

$$
A_{k}=\bigcup_{i \in I_{k}}\left[2^{i-k-1}, 2^{i-k}\right) \cap \omega .
$$

Note that $\nu\left(A_{k}\right) \leq 2^{-k}$. Indeed, if $i \in I_{k}$, then $A_{k} \cap\left[2^{i-k}, 2^{i}\right)=\emptyset$, so $\left|A_{k} \cap 2^{i}\right| / 2^{-i} \leq$ $2^{-k}$.

Now we check that, given a set $B$ with $\nu(B)<1 / 4$, we have $\nu\left(A_{k} \backslash B\right)>0$ for infinitely many $k$. Let

$$
J=\left\{i:\left|B \cap\left[2^{i-1}, 2^{i}\right)\right| \leq 2^{i-2}\right\} .
$$

Then $J \in \mathcal{U}$, since otherwise we would have $\nu(B) \geq 1 / 4$.

It follows from the assumption on $\mathcal{U}$ that $J+k \in \mathcal{U}$ for infinitely many $k$. Fix a number $k$ with this property, put $K=(J+k) \cap I_{k}$, and consider any $i \in K$. Since $i \in I_{k}$ and $i-k \in J$, we have

$$
A_{k} \supseteq\left[2^{i-k-1}, 2^{i-k}\right) \cap \omega, \quad\left|B \cap\left[2^{i-k-1}, 2^{i-k}\right)\right| \leq 2^{i-k-2} .
$$

Hence

$$
\begin{gathered}
\left|\left(A_{k} \backslash B\right) \cap 2^{i}\right| \geq\left|\left(A_{k} \backslash B\right) \cap\left[2^{i-k-1}, 2^{i-k}\right)\right| \geq 2^{i-k}-2^{i-k-1}-2^{i-k-2}=2^{i-k-2} ; \\
\frac{\left|\left(A_{k} \backslash B\right) \cap 2^{i}\right|}{2^{i}} \geq 2^{-(k+2)},
\end{gathered}
$$

whenever $i \in K \in \mathcal{U}$. Hence $\nu\left(A_{k} \backslash B\right)>0$. Therefore, the sequence $A_{4}, A_{4} \cup A_{5}, \ldots$ witnesses that $\nu$ fails to have $\mathbf{A P}$ (null).

In order to get a density without property $\mathbf{A P}$ (null), it is now sufficent to find an ultrafilter $\mathcal{U}$ such that for every $A \in \mathcal{U}$ there is a $k>0$ with $A+k \in \mathcal{U}$. D. Fremlin pointed out that the existence of an ultrafilter with this property may be quickly derived form Glazer's theorem on idempotent ultrafilters (see e.g. [9], section 15). This suggested to us the following straightforward argument.

The formula $\phi(\mathcal{X})=\{A: A+1 \in \mathcal{X}\}$ defines a continuous mapping $\phi: \beta \omega \backslash \omega \rightarrow$ $\beta \omega \backslash \omega$. By compactness, there is a minimal $\phi$-invariant closed and nonempty subset $S$ of $\beta \omega \backslash \omega$. We check that any $\mathcal{U} \in S$ has the required property. Indeed, the orbit $\left\{\phi^{k}(\mathcal{U}): k \geq 1\right\}$ must be dense in $S$, since its closure is $\phi$-invariant. Therefore, given $A \in \mathcal{U}$, there is a $k \geq 1$ such that the ultrafilter $\phi^{k}(\mathcal{U})$ is in the closed and open set defined by $A$. Hence $A \in \phi^{k}(\mathcal{U})$, so $A+k \in \mathcal{U}$, and we are done.

\section{ACKNOWLEDGEMENTS}

We would like to thank the referee for a careful reading of the first version of this paper, and for several valuable comments. Thanks are also due to David Fremlin for sending us his result, and for his kind permission to include it here.

\section{REFERENCES}

[1] K.P.S. Bhaskara Rao, M. Bhaskara Rao, Theory of charges, Academic Press (1983). MR 86f:28006

[2] R.C. Buck, Generalized asymptotic density, Amer. J. Math. 75 (1953), 335-346. MR 14:854t

[3] R. Frankiewicz, S. Shelah, P. Zbierski, On closed P-sets with ccc in the space $\omega^{*}$, J. Symbolic Logic 58 (1993), 1171-1176. MR 95c:03125]

[4] S. Gangopadhyay, On the completeness of $L_{p}$ spaces over a charge, Colloq. Math. 58 (1990), 291-300. MR 91h:46049 
3320 A. BLASS, R. FRANKIEWICZ, G. PLEBANEK, AND C. RYLL-NARDZEWSKI

[5] S. Gangopadhyay, B.V. Rao, Completeness of $L_{p}$ spaces over finitely additive measures, Colloq. Math. 80 (1999), 83-95. MR 2000f:28006

[6] D. Maharam, Finitely additive measures on the integers, Sankhya Ser. A 38 (1976), 44-59. MR 57:12810

[7] A.H. Mekler, Finitely additive measures on $N$ and the additive property, Proc. Amer. Math. Soc. 92 (1984), 439-444. MR 86j:28003

[8] S. Shelah, Proper forcing, Lecture Notes in Mathematics 940, Springer-Verlag, Berlin 1982. MR 84h:03002

[9] S. Todorcevic, Topics in topology, Springer (1997), Berlin. MR 98g:54002

Department of Mathematics, University of Michigan, Ann Arbor, Michigan 481091109

E-mail address: ablass@math.lsa.umich.edu

Institute of Mathematics, Polish Academy of Sciences, 00-950 Warsaw, Poland

E-mail address: rf@impan.gov.pl

Institute of Mathematics, University of WrocŁaW, Pl. Grunwaldzki 2/4, 50-218 Wroceaw, Poland

E-mail address: grzes@math.uni.wroc.pl

Institute of Mathematics, Wroceaw Technical University and Institute of Mathematics, Polish Academy of Sciences, 51-617 Wroceaw, Poland

E-mail address: crn@graf.im.pwr.wroc.pl 